\title{
Search Functionality Within the Electronic Health Record and Health Information
}

\section{Exchange Databases}

\author{
Brandon Gregory ${ }^{1}$, Jordan Hill, Titus Schleyer ${ }^{1,2,3}$ \\ ${ }^{1}$ Indiana University School of Medicine; ${ }^{2}$ Indiana University School of Medicine, Department of \\ Biomedical Informatics; ${ }^{3}$ Regenstrief Institute, Center for Biomedical Informatics
}

\section{Background and Hypothesis:}

In the US today, over $95 \%$ of healthcare institutions operate using the electronic health record (EHR). While proven to be a substantial improvement to medical practice, the substantial amount of retained information within those records has made searching the EHR for relevant material difficult and too time consuming. We hypothesize that by providing a search function within the EHR with added capability of collaborative filtration, physicians will be better able to retrieve important patient information and thus provide more efficient care.

\section{Project Methods:}

Emergency Department physicians of Sidney \& Lois Eskenazi Hospital and Indiana University Health Hospital were recruited to partake in this study based on their use and familiarity of the EHR Cerner and/or Health Information Exchange (HIE) CareWeb Search function. Participants filled out a pre-interview, Likert-scale questionnaire to determine their general impressions of search functions and the frequency with which they were used. Additional insight was obtained during an interview focusing on participants' previous experiences searching within the EHR/HIE. Participants were then shown a mock-up of potential collaborative filtering integration into CareWeb in order to collect opinions regarding the feature's usability/practicality, display/format, and a number of suggested terms.

\section{Results:}

From the pilot study, current challenges that limit clinician search function use include limited time in clinician workflow, information overload, and inaccurate results. Clinicians are more likely to conduct searches when treating patients who have limited medical history, complex histories, known recent visitations, and/or who have been seen at other institutions. Participants demonstrated interest in a collaborative filtration search feature; they expressed a preference to have the feature recommend five related search terms.

\section{Potential Impact:}

The data from this study aims to refine the way healthcare providers search within the EHR/HIE. This will allow healthcare providers to more efficiently extract relevant patient information for improved healthcare delivery and proficient clinician workflow. 